\title{
Padrões de consumo alimentar em crianças menores de dois anos no Brasil: Pesquisa Nacional de Saúde, 2013
}

\author{
Food consumption patterns in children under two years of age in \\ Brazil: National Health Survey, 2013
}

Thaynã Ramos Flores (https://orcid.org/0000-0003-0098-1681) ${ }^{1}$

Rosália Garcia Neves (https://orcid.org/0000-0001-6798-9130) ${ }^{2}$

Andrea Wendt (https://orcid.org/0000-0002-4640-2254) ${ }^{1}$

Caroline dos Santos Costa (https://orcid.org/0000-0002-3522-1546) ${ }^{1}$

Andréa Dâmaso Bertoldi (https://orcid.org/0000-0002-4680-3197) ${ }^{1}$

Bruno Pereira Nunes (https://orcid.org/0000-0002-4496-4122) ${ }^{3}$

${ }^{1}$ Programa de PósGraduação em

Epidemiologia, Universidade Federal de Pelotas (UFPel). R. Marechal Deodoro 1160, Centro. 96020-220 Pelotas RS Brasil. floresrthayna@gmail.com $23^{\mathrm{a}}$ Coordenadoria Regional de Saúde. Pelotas RS Brasil.

${ }^{3}$ Faculdade de Enfermagem, UFPel. Pelotas RS Brasil.

\begin{abstract}
The scope of this paper was to identify patterns of food consumption and associated factors among children under two years of age in $\mathrm{Bra}$ zil. It involved a cross-sectional, population-based study using data from the National Health Survey conducted in 2013. Food consumption was measured by dichotomous questions, listing the food ingested solely on the day prior to the interview. The patterns were identified through principal component analysis. Poisson regression was used to obtain a crude and adjusted prevalence ratio. The sample design effect was considered in the analyses that were stratified according to age. Among the 5,052 children, three patterns were identified, being defined as: a "healthy food consumption pattern," a "milk consumption pattern" and an "unhealthy food consumption pattern." There was an association between the patterns in each age group, mainly related to skin color, gender, schooling of the head of the family, area of residence and region. A quarter of the children from 12 to 23 months consumed soft drinks, more than a third drank artificial juices and almost half ate candies the day before the interview. Three dietary patterns were identified, besides observing early food ingestion among children under six months of age.

Key words Dietary habits, Eating behavior, Children, Principal component analysis
\end{abstract}

Resumo Objetivou-se identificar os padrões de consumo alimentar e os fatores associados em crianças menores de dois anos no Brasil. Estudo transversal, de base populacional, utilizando dados da Pesquisa Nacional de Saúde realizada em 2013. O consumo alimentar foi mensurado por questôes dicotômicas, listando os alimentos, considerando apenas o dia anterior à entrevista. Os padrões foram identificados por meio de análise de componentes principais. Utilizou-se regressão de Poisson para obtenção de razão de prevalência bruta e ajustada. Considerou-se o efeito de delineamento amostral nas análises que foram estratificadas de acordo com a idade. Dentre as 5.052 crianças, foram identificados três padrões, sendo denominados como: "padrão de consumo de alimentos saudáveis", "padrão de consumo de leites" $e$ "padrão de consumo de alimentos não saudáveis". Observou-se associação entre os padrões em cada faixa etária, principalmente com cor da pele, sexo, escolaridade do chefe da família, zona de residência e região. Um quarto das crianças de 12 a 23 meses ingeriu refrigerantes, mais de um terço sucos artificiais e quase metade doces no dia anterior à entrevista. Foram identificados três padrões alimentares, além de observar introdução alimentar precoce em crianças menores de seis meses de idade.

Palavras-chave Hábitos alimentares, Comportamento alimentar, Crianças, Análise de componentes principais 


\section{Introdução}

A alimentação de crianças menores de 24 meses passa por diversas mudanças, inicialmente sendo recomendado, até os seis meses de idade, somente o consumo leite materno, seguido da introdução alimentar após este período ${ }^{1-3}$. No entanto, introduzir alimentos nessa idade não se restringe somente ao fato de alimentar a criança, mas sim, de oferecer uma alimentação complementar que seja livre de alimentos industrializados e ultraprocessados, o que configura em uma alimentação complementar saudável ${ }^{3,4}$.

O padrão alimentar é caracterizado pelo conjunto de alimentos que são habitualmente consumidos pelos indivíduos ${ }^{5,6}$. O conhecimento de padrões alimentares de uma população é importante para delinear ações voltadas à educação alimentar, com o objetivo de reduzir os danos causados por uma alimentação não saudável ${ }^{7}$. Ainda, as crianças são os principais alvos de propagandas vinculadas aos alimentos considerados não saudáveis e, por isso, compreender os padrões adotados ainda na infância mostra-se relevante ${ }^{8}$.

Alguns estudos avaliavam, de forma tradicional, o padrão alimentar a partir da disponibilidade de macro e micronutrientes e do conteúdo energético da dieta ${ }^{7,9,10}$. No entanto, a Organização Mundial da Saúde (OMS) instituiu que o consumo alimentar pode ser analisado utilizando os alimentos e não propriamente os nutrientes presentes neles ${ }^{11}$. Sendo assim, estudos com questionários que remetam ao consumo alimentar habitual podem identificar padrões alimentares.

A maior parte dos estudos envolvendo padrão alimentar de crianças incluem somente aquelas de 2 a 5 anos de idade ou em idade escolar ${ }^{6,7,12,13}$, não sendo possível estabelecer o padrão alimentar de crianças menores de 24 meses. Alguns poucos estudos abordam crianças de 12 meses ou mais ${ }^{14,15}$. Mesmo com todos esforços para o aumento das prevalências de aleitamento materno, cabe destacar que estudo recente identificou que apenas 20,6\% das crianças menores de seis meses de idade haviam recebido exclusivamente leite materno no dia anterior à entrevista ${ }^{16}$. Estudos sobre o padrão alimentar de crianças menores de 24 meses são necessários para contribuir com as políticas que visam garantir uma melhor nutrição na infância ${ }^{17}$. Nesse sentido, o objetivo deste estudo foi identificar os padrões de consumo alimentar e os fatores associados aos mesmos em crianças menores de dois anos no Brasil.

\section{Métodos}

Estudo transversal, de base populacional, utilizando dados da Pesquisa Nacional de Saúde (PNS) realizada em 2013 no Brasil. A pesquisa foi realizada a partir de uma parceria entre o Instituto Brasileiro de Geografia e Estatística (IBGE) e o Ministério da Saúde (MS). A amostra é representativa de moradores de domicílios permanentes, localizados em área urbana ou rural, das cinco grandes regiões geográficas, 27 Unidades da Federação (UF), capitais e municípios.

A amostragem foi realizada em três estágios, sendo o primeiro a seleção de setores censitários, seguido dos domicílios e, por último, os indivíduos. Os respondentes eram os moradores adultos (18 anos ou mais) e a as mães ou responsáveis responderam às questões referentes aos menores de dois anos de idade. Maior detalhamento do processo de amostragem encontra-se no artigo metodológico da $\mathrm{PNS}^{18}$.

A coleta dos dados, por meio de computadores de mão (personal digital assistance [PDA]), foi realizada por entrevistadores treinados. O questionário da PNS foi constituído de três partes. Inicialmente, foram coletadas variáveis do domicílio, sendo esse bloco respondido pela pessoa responsável pelo domicílio ou aquela apta a responder essas informações no momento da entrevista. A segunda parte refere-se às características gerais, respondida por todos os moradores do domicílio, incluindo questões sobre nível de educação, trabalho, rendimento, deficiências, cobertura de plano de saúde, utilização dos serviços de saúde, saúde do idoso, cobertura de mamografia e características de crianças menores de dois anos de idade, sendo essa seção utilizada no presente estudo. Na terceira parte, questões sobre outras características (trabalho e apoio social, percepção do estado de saúde, acidentes e violências, estilos de vida, doenças crônicas, saúde da mulher, atendimento pré-natal, saúde bucal e atendimento médico) foram direcionadas a um morador adulto selecionado aleatoriamente. Essa seção do questionário só poderia ser respondida pelo morador selecionado.

O consumo alimentar foi avaliado por meio do questionamento: "Você pode me dizer quais destes alimentos < a criança $>$ tomou ou comeu desde ontem de manhã até hoje de manhã?", sendo listados os seguintes alimentos e bebidas: leite materno, outros tipos de leite, mingau, legumes e verduras, frutas, carnes e ovos, batata, cereais, feijão, sucos artificias, bolachas, refrigerantes e doces. 
Os padrões alimentares foram identificados por meio de análise de componentes principais $(\mathrm{ACP})^{19}$, que permite que os grupos alimentares sejam combinados com base no grau de correlação entre eles. Primeiramente, foi realizada análise sem restrição quanto ao número de fatores a serem retidos, e ainda, utilizou-se a rotação ortogonal varimax para obter padrões não correlacionados entre si e melhorar a interpretação dos dados. O número de padrões a serem extraídos foi definido com base nos critérios de autovalores maiores do que a unidade e no gráfico screeplot, no qual os pontos de maior declive indicam o número de fatores a ser considerado na análi$\mathrm{se}^{20}$. Posteriormente a essas análises, o modelo foi construído fixando o número de padrões a serem retidos, sendo identificados três tipos de padrões alimentares. Os grupos que contribuíram para a caracterização de cada padrão foram aqueles com cargas fatoriais $\geq 0,3$ ou $\leq-0,3$. Os padrões foram nomeados com base nas características dos itens retidos como: "alimentos saudáveis", "leites" e "alimentos não saudáveis"

As variáveis independentes foram o sexo (masculino/feminino) e a cor da pele da criança (branca, preta, parda), escolaridade do chefe da família (sem instrução; ensino fundamental; ensino médio; ensino superior), índice de riqueza em quintis (com base em propriedade de banheiro dentro de casa, automóvel, motocicleta, geladeira, máquina de lavar roupas, aparelho de DVD, televisão, telefone fixo, computador e micro-ondas) (Critério de Classificação Econômica Brasil, http://www.abep.org), zona de moradia (urbana; rural) e região geográfica (Norte; Nordeste; Centro-Oeste; Sudeste; Sul). Para análises que compararam grupos, a região Sudeste foi tratada como referência por ser a terceira região que concentra maior $\mathrm{N}$ amostral e, também, porque o Nordeste e Norte do país ainda concentram a maior taxa de natalidade.

As análises foram realizadas no programa Stata ${ }^{\circledR}$ 15.0. Inicialmente, realizou-se a descrição dos alimentos consumidos no dia anterior à entrevista e das variáveis independentes, obtendose suas prevalências. A associação entre as variáveis independentes e cada padrão alimentar foi avaliada por meio de regressão de Poisson com ajuste robusto da variância ${ }^{21}$. Foi realizada análise ajustada para as variáveis socioeconômicas e demográficas, sendo mantidas no modelo final somente aquelas que apresentaram um valor de $p<0,20$. Associações que apresentaram valor de $\mathrm{p}<0,05$ foram consideradas estatisticamente significativas. Todas as análises foram realizadas uti- lizando o comando svy para considerar o efeito de delineamento amostral do estudo e estratificadas de acordo com a idade da criança (0-5 meses e 29 dias; 6-11 meses e 29 dias e $12-23$ meses e 29 dias de idade).

O projeto foi submetido à Comissão Nacional de Ética em Pesquisa e aprovado. Todos os participantes assinaram o termo de consentimento livre e esclarecido, garantindo o sigilo dos dados.

\section{Resultados}

A amostra deste estudo foi de 4.863 crianças. Aproximadamente metade da amostra era composta por crianças entre 12 e 23 meses e cerca de um terço pertencia à região Norte e um terço à região Nordeste. Pouco mais da metade era de cor da pele parda (51,7\%). Quanto à escolaridade do chefe da família, houve predomínio do nível de ensino fundamental completo $(36,3 \%)$. Em relação à posse de bens, 27,3\% das crianças pertenciam ao $2^{\circ}$ quinto da distribuição e $79,1 \%$ residia na zona urbana (Tabela 1 ).

A Figura 1 apresenta a distribuição de todos os alimentos ingeridos no dia anterior à entrevista estratificada pela idade das crianças. Entre as crianças menores de seis meses de idade, os alimentos mais consumidos foram o leite materno seguido por mingau e outros leites. Entre as crianças de 12 a 23 meses observou-se maior prevalência de consumo de bolachas, cereais, batata, frutas, carnes e ovos, feijão e legumes e verduras, e menor de leite materno. Cabe destacar que, do total da amostra, 16,2\% ingeriu refrigerantes, $25,6 \%$ sucos artificiais e $29,6 \%$ consumiu doces no dia anterior à entrevista, sendo essas prevalências maiores no estrato de crianças de 12 a 23 meses de idade.

Três padrões de consumo alimentar foram identificados. Os alimentos com cargas fatoriais $\geq 0,3$ estão destacados. O padrão de "alimentos saudáveis" caracterizou-se pelo consumo frutas, legumes e verduras, carnes e ovos, batata/mandioca, cereais, bolachas e feijão. O padrão de "leites" foi caracterizado pelo consumo de leite materno, outros leites e mingau. E, ainda, o padrão de "alimentos não saudáveis" consistiu em consumo de doces, refrigerantes e sucos artificiais (Tabela 2).

A Tabela 3 apresenta a análise bruta dos três padrões de consumo alimentar de acordo com as variáveis independentes, estratificada pela faixa etária das crianças menores de dois anos. $\mathrm{Na}$ análise ajustada, entre as crianças menores de seis meses de idade encontrou-se associação estatis- 
Tabela 1. Descrição das características da amostra de crianças menores de dois anos de idade. Pesquisa Nacional de Saúde, Brasil, 2013 ( $\mathrm{n}=5.052$ ).

\begin{tabular}{|c|c|c|c|c|}
\hline Variáveis & $\begin{array}{c}\mathbf{0} \text { até }<6 \\
\text { meses } \\
\mathrm{N}(\%)^{\star} \\
(\mathrm{n}=1.414)\end{array}$ & $\begin{array}{c}6 \text { até }<12 \\
\text { meses } \\
N(\%)^{\star} \\
(\mathrm{n}=1.273)\end{array}$ & $\begin{array}{c}12 \text { até }<24 \\
\text { meses } \\
N(\%)^{\star} \\
(n=2.365)\end{array}$ & $\begin{array}{c}\text { Total da } \\
\text { amostra } \\
N(\%)^{\star} \\
(n=5.052)\end{array}$ \\
\hline \multicolumn{5}{|l|}{ Sexo } \\
\hline Masculino & $704(49,8)$ & $621(48,8)$ & $1.213(51,3)$ & $2.538(50,2)$ \\
\hline Feminino & $710(50,2)$ & $652(51,2)$ & $1.152(48,7)$ & $2.514(49,8)$ \\
\hline \multicolumn{5}{|l|}{ Cor da pele ${ }^{\star *}$} \\
\hline Branca & $639(45,8)$ & $531(42,0)$ & $984(42,1)$ & $2.154(43,1)$ \\
\hline Preta & $66(4,7)$ & $73(5,8)$ & $119(5,1)$ & $258(5,2)$ \\
\hline Parda & $689(49,5)$ & $660(52,2)$ & $1.233(52,8)$ & $2.582(51,7)$ \\
\hline \multicolumn{5}{|l|}{ Escolaridade chefe da família } \\
\hline Sem instrução & $209(14,8)$ & $167(13,1)$ & $353(14,9)$ & $729(14,4)$ \\
\hline Ensino fundamental & $508(35,9)$ & $451(35,4)$ & $874(37,0)$ & $1.833(36,3)$ \\
\hline Ensino médio & $474(33,5)$ & $441(34,6)$ & $795(33,6)$ & $1.710(33,9)$ \\
\hline Superior & $223(15,8)$ & $214(16,8)$ & $343(14,5)$ & $780(15,4)$ \\
\hline \multicolumn{5}{|l|}{ Posse de bens } \\
\hline $1^{\circ}$ quintil (mais pobre) & $335(23,7)$ & $294(23,1)$ & $595(25,2)$ & $1.224(24,2)$ \\
\hline $2^{\circ}$ quintil & $393(27,8)$ & $357(28,0)$ & $631(26,7)$ & $1.381(27,3)$ \\
\hline $3^{\circ}$ quintil & $253(17,9)$ & $213(16,7)$ & $437(18,5)$ & $903(17,9)$ \\
\hline $4^{\circ}$ quintil & $228(16,1)$ & $221(17,4)$ & $381(16,1)$ & $830(16,4)$ \\
\hline $5^{\circ}$ quintil (mais rico) & $205(14,5)$ & $188(14,8)$ & $321(13,6)$ & $714(14,1)$ \\
\hline \multicolumn{5}{|l|}{ Zona de residência } \\
\hline Rural & $286(20,2)$ & $241(18,9)$ & $527(22,3)$ & $1.054(20,9)$ \\
\hline Urbana & $1.128(79,8)$ & $1.032(81,1)$ & $1.838(77,7)$ & $3.998(79,1)$ \\
\hline \multicolumn{5}{|l|}{ Região } \\
\hline Sudeste & $258(18,3)$ & $199(15,6)$ & $431(18,2)$ & $888(17,6)$ \\
\hline Nordeste & $422(30,0)$ & $354(27,8)$ & $715(30,3)$ & $1.493(29,6)$ \\
\hline Centro-Oeste & $166(11,7)$ & $173(13,6)$ & $284(12,0)$ & $623(12,3)$ \\
\hline Norte & $410(29,0)$ & $419(32,9)$ & $699(29,6)$ & $1.528(30,3)$ \\
\hline Sul & $156(11,0)$ & $128(10,1)$ & $236(10,0)$ & $520(10,3)$ \\
\hline
\end{tabular}

ticamente significativa entre padrão de alimentos não saudáveis e cor da pele preta com maior probabilidade de consumo de alimentos deste padrão [RP=1,87 (IC95\% 1,28; 2,74) $\mathrm{p}=0,005]$, escolaridade do chefe da família "nível superior" com menor probabilidade de consumo $[\mathrm{RP}=0,52$ (IC95\% 0,32; 0,85) p=0,006] e região Nordeste, Centro-Oeste e Norte $(\mathrm{p}<0,001)$. Ainda, entre os menores de seis meses de idade, encontrou-se associação com posse de bens onde crianças pertencentes ao $3^{\circ}$ e $4^{\circ}$ quintil apresentaram maior probabilidade de consumir alimentos que compõem o padrão saudável [RP=1,39 (IC95\% 1,13; 1,71); $\mathrm{RP}=1,31$ (IC95\% 1,05; 1,63) p=0,006], respectivamente. Em crianças de 6 a 12 meses não foram observadas associações estatisticamente significativas com o padrão de alimentos não saudáveis.
Já nos de 12 a 23 meses de idade, observou-se que crianças pertencentes a famílias cujo chefe tinha ensino médio ou completo $(\mathrm{p}<0,001)$ e que residiam em zona urbana $(\mathrm{p}=0,02)$ apresentaram maior probabilidade de consumo de alimentos do padrão saudável. Já as crianças que residiam na região Norte e Nordeste apresentaram menor probabilidade de consumo de alimentos do padrão saudável $(\mathrm{p}<0,001)$. Nesta mesma faixa etária, identificou-se que crianças do sexo feminino $[\mathrm{RP}=1,05$ (IC95\% 1,02; 1,09) $\mathrm{p}=0,002]$ e de cor da pele preta $[\mathrm{RP}=1,11$ (IC95\% 1,$05 ; 1,18$ ) $\mathrm{p}=0,002]$ tiveram maior probabilidade de consumir alimentos do padrão não saudável, enquanto que as pertencentes a região Norte e Nordeste tiveram menor probabilidade de ingerir alimentos do mesmo padrão $(\mathrm{p}<0,001)$ (Tabela 4$)$. 


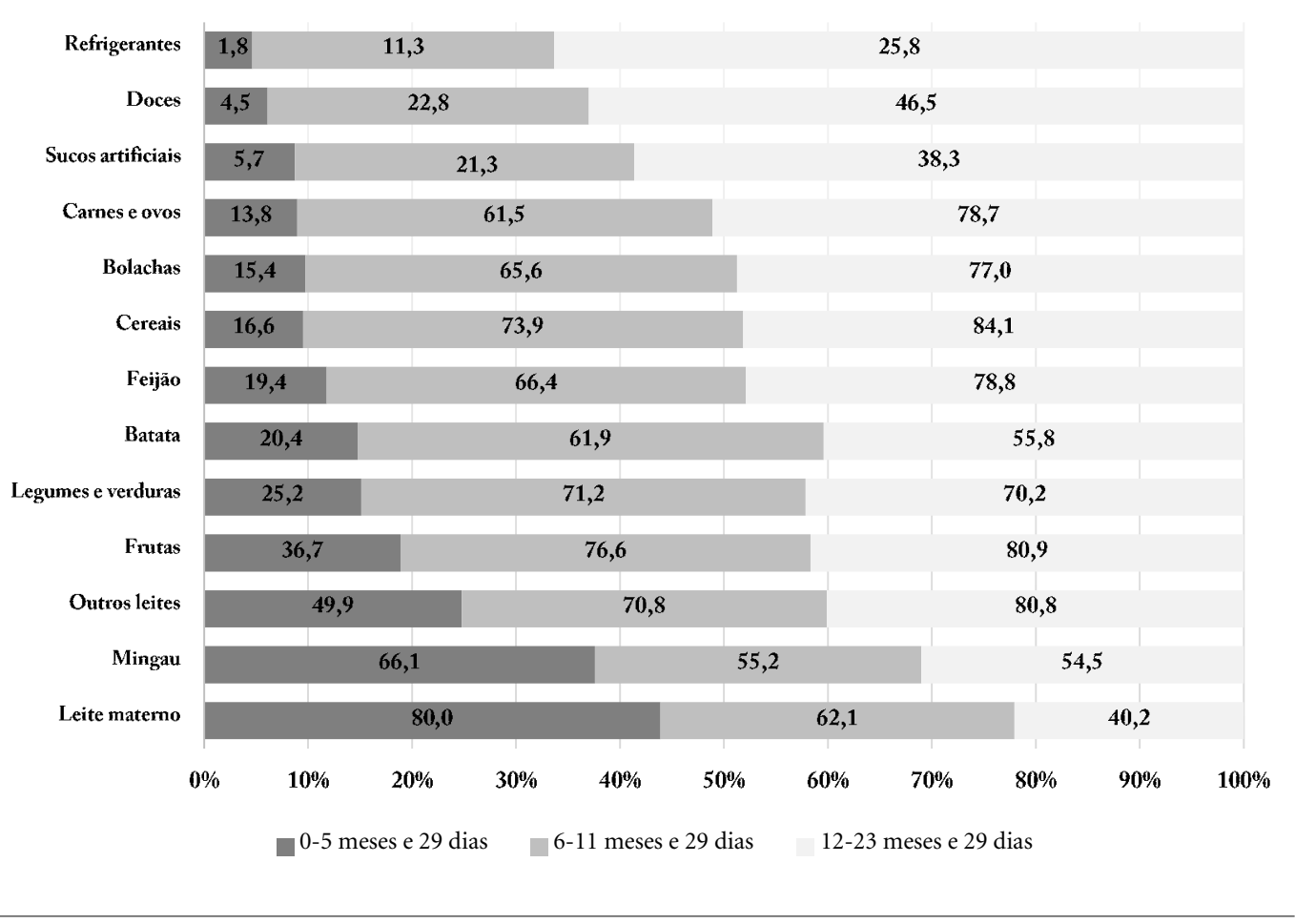

Figura 1. Prevalência de cada alimento consumido no dia anterior à entrevista, estratificada pela faixa etária das crianças menores de dois anos. Pesquisa Nacional de Saúde, $2013(\mathrm{n}=4.863)$.

\section{Discussão}

Neste estudo, foram identificados três padrões de consumo alimentar de crianças menores de 24 meses de idade: "alimentos saudáveis", "leites" e "alimentos não saudáveis". Entre as crianças menores de seis meses de idade, após os ajustes, observou-se associação entre o padrão de alimentos não saudáveis com a cor da pele preta, maior escolaridade do chefe da família e as regiões Norte, Nordeste e Centro-Oeste em comparação ao Sudeste. Também após os ajustes, entre as crianças de 6 a 11 meses, observou-se associação de consumo do padrão de leites com zona de residência. Já para as de 12 a 23 meses, observou-se associação entre padrão de consumo de alimentos não saudáveis com sexo, cor da pele e região. Ainda, cabe destacar que entre as crianças de 12 a 23 meses, cerca de um quarto ingeriu refrigerantes, mais de um terço sucos artificiais e quase metade doces.

A informação sobre padrões alimentares em crianças ainda é incipiente. Estudo realizado por Souza et al. ${ }^{15}$, utilizando a mesma técnica de análise por componentes principais, identificou mais padrões de alimentação do que os observados no presente estudo. Crianças de um a seis anos de idade apresentaram cinco padrões alimentares, sendo eles: "vegetais", "tradicional" (pão, margarina/margarina, arroz/massa, café, açúcar), "guloseimas e embutidos", "lanches" (laticínios, achocolatados, biscoitos e sucos) e "frutas". Essa diferença pode ser explicada pela diferente faixa etária entre os estudos já que crianças mais velhas tendem a apresentar padrão de consumo alimentar mais diversificado. Já no estudo de Matos et al. ${ }^{6}$, também foram identificados três padrões alimentares e, ainda, observou-se que o leite materno teve seu consumo diminuído no padrão de alimentação de crianças menores de seis meses de idade, possivelmente por consequência de maior consumo de leite de vaca ${ }^{6}$.

Mesmo com todas evidências e forte recomendação de que o aleitamento materno deve ser o alimento exclusivo até os seis meses de idade das crianças, outro estudo realizado com dados também da PNS identificou que aproximadamente $20 \%$ das crianças receberam exclusivamente leite materno nesse período de vida (zero a 


\begin{tabular}{|c|c|c|c|c|c|c|c|c|c|c|}
\hline \multirow[t]{20}{*}{ 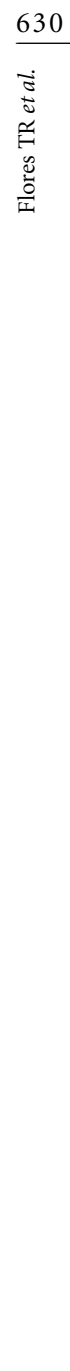 } & \multicolumn{10}{|c|}{$\begin{array}{l}\text { Tabela 2. Distribuição das cargas fatoriais dos padrões de consumo alimentar, estratificada pela faixa etária das } \\
\text { crianças menores de dois anos. Pesquisa Nacional de Saúde, } 2013(\mathrm{n}=4.863) \text {. }\end{array}$} \\
\hline & \multirow[b]{3}{*}{ Alimentos } & \multicolumn{9}{|c|}{ Padrões de consumo alimentar } \\
\hline & & \multicolumn{3}{|c|}{0 até $<6$ meses } & \multicolumn{3}{|c|}{6 até $<12$ meses } & \multicolumn{3}{|c|}{12 até $<24$ meses } \\
\hline & & $\begin{array}{l}\text { Alimentos } \\
\text { saudáveis }\end{array}$ & Leites & $\begin{array}{l}\text { Alimentos } \\
\text { não } \\
\text { saudáveis }\end{array}$ & $\begin{array}{l}\text { Alimentos } \\
\text { saudáveis }\end{array}$ & Leites & $\begin{array}{c}\text { Alimentos } \\
\text { não } \\
\text { saudáveis }\end{array}$ & $\begin{array}{c}\text { Alimentos } \\
\text { saudáveis }\end{array}$ & Leites & $\begin{array}{l}\text { Alimentos } \\
\text { não } \\
\text { saudáveis }\end{array}$ \\
\hline & $\begin{array}{l}\text { Leite } \\
\text { materno }\end{array}$ & 0,02 & $-0,55$ & $-0,15$ & $-0,17$ & $-0,46$ & 0,27 & $-0,29$ & $-0,49$ & 0,27 \\
\hline & Outros leites & 0,06 & 0,59 & 0,20 & 0,20 & 0,45 & $-0,33$ & 0,34 & 0,48 & $-0,24$ \\
\hline & Mingau & 0,07 & 0,41 & 0,29 & 0,09 & 0,50 & $-0,24$ & 0,11 & 0,38 & $-0,20$ \\
\hline & $\begin{array}{l}\text { Legumes e } \\
\text { verduras }\end{array}$ & 0,33 & 0,13 & 0,13 & 0,36 & $-0,22$ & $-0,20$ & 0,39 & $-0,29$ & $-0,06$ \\
\hline & $\begin{array}{l}\text { Carnes e } \\
\text { ovos }\end{array}$ & 0,35 & $-0,03$ & $-0,20$ & 0,38 & $-0,17$ & 0,03 & 0,36 & $-0,15$ & 0,13 \\
\hline & Frutas & 0,26 & 0,23 & -0.14 & 0,32 & $-0,09$ & $-0,19$ & 0,32 & $-0,24$ & $-0,07$ \\
\hline & Batata & 0,35 & 0,08 & $-0,29$ & 0,37 & $-0,23$ & $-0,14$ & 0,34 & $-0,23$ & 0,05 \\
\hline & Cereais & 0,36 & 0,01 & $-0,07$ & 0,36 & $-0,07$ & 0,06 & 0,34 & $-0,05$ & 0,14 \\
\hline & Feijão & 0,36 & $-0,009$ & $-0,14$ & 0,35 & $-0,17$ & 0,06 & 0,33 & $-0,15$ & 0,10 \\
\hline & $\begin{array}{l}\text { Sucos } \\
\text { artificiais }\end{array}$ & 0,23 & $-0,10$ & 0,43 & 0,14 & 0,24 & 0,43 & 0,02 & 0,25 & 0,43 \\
\hline & Refrigerantes & 0,26 & $-0,22$ & 0,49 & 0,13 & 0,25 & 0,49 & $-0,03$ & 0,20 & 0,52 \\
\hline & Bolachas & 0,34 & $-0,02$ & 0,06 & 0,29 & 0,08 & 0,16 & 0,24 & 0,09 & 0,26 \\
\hline & Doces & 0,27 & $-0,20$ & 0,42 & 0,22 & 0,18 & 0,44 & 0,05 & 0,22 & 0,50 \\
\hline & $\begin{array}{l}\text { Número de } \\
\text { itens }\end{array}$ & 6 & 3 & 3 & 6 & 3 & 4 & 7 & 3 & 3 \\
\hline & Eigennvalues & 4,46 & 1,78 & 1,29 & 3,36 & 1,44 & 1,60 & 2,84 & 1,23 & 1,77 \\
\hline & $\begin{array}{l}\text { \% variância } \\
\text { acumulada }\end{array}$ & $34,3 \%$ & $48,0 \%$ & $58,0 \%$ & $25,8 \%$ & $49,2 \%$ & $38,2 \%$ & $21,8 \%$ & $44,9 \%$ & $35,4 \%$ \\
\hline
\end{tabular}

seis meses de idade) ${ }^{16}$. É importante ressaltar que no presente estudo o padrão chamado de "saudável"4 se refere aos alimentos que compõem esse grupo e não que sejam os apropriados ou preconizados para as crianças menores de seis meses, como por exemplo, a inclusão de bolacha, sendo que nessa faixa etária é recomendado o consumo de leite materno exclusivo ${ }^{1,3}$.

Com relação aos fatores associados aos padrões alimentares, crianças de cor da pele preta tiveram maior probabilidade de aderir ao padrão de consumo de alimentos não saudáveis. Considerando que a cor da pele é um marcador de nível econômico, ressalta-se que, em parte, esse achado reflete a transição nutricional que possibilitou a maior disponibilidade de alimentos considerados não saudáveis para a população no geral ${ }^{22}$ que, em muitas vezes, busca este tipo de alimentação mesmo que ainda tenha outros alimentos que componham uma alimentação saudáve ${ }^{23}$. Assim, as crianças que pertencem a famílias cuja alimentação se baseia em uma dieta restrita em alimentos saudáveis estão suscetíveis aos mesmos hábitos alimentares ${ }^{24,25}$. E mesmo entre as menores de seis meses que deveriam estar recebendo leite materno exclusivamente, conforme preconizado pela $\mathrm{OMS}^{1,3}$, acabam aderindo mais ao padrão não saudável o que é ressaltado pelos resultados de Victora e colaboradores que encontraram maior prevalência de aleitamento materno entre as crianças pertencentes aos estratos mais ricos da população ${ }^{26}$.

Ainda, foi possível observar que crianças pertencentes à região Norte tiveram menor probabilidade de consumo de alimentos do padrão considerado não saudável, o que poderia ser explicado, em parte, pela disponibilidade das mães em amamentar exclusivamente e por mais tempo, considerando o contexto e os aspectos culturais dessa região $0^{27}$. Outro estudo que avaliou consumo de leite materno, encontrou prevalência de $22 \%$ de aleitamento materno exclusivo na região Norte $^{16}$, sendo a terceira prevalência mais alta. Além disso, esse achado pode ser explicado pelo 


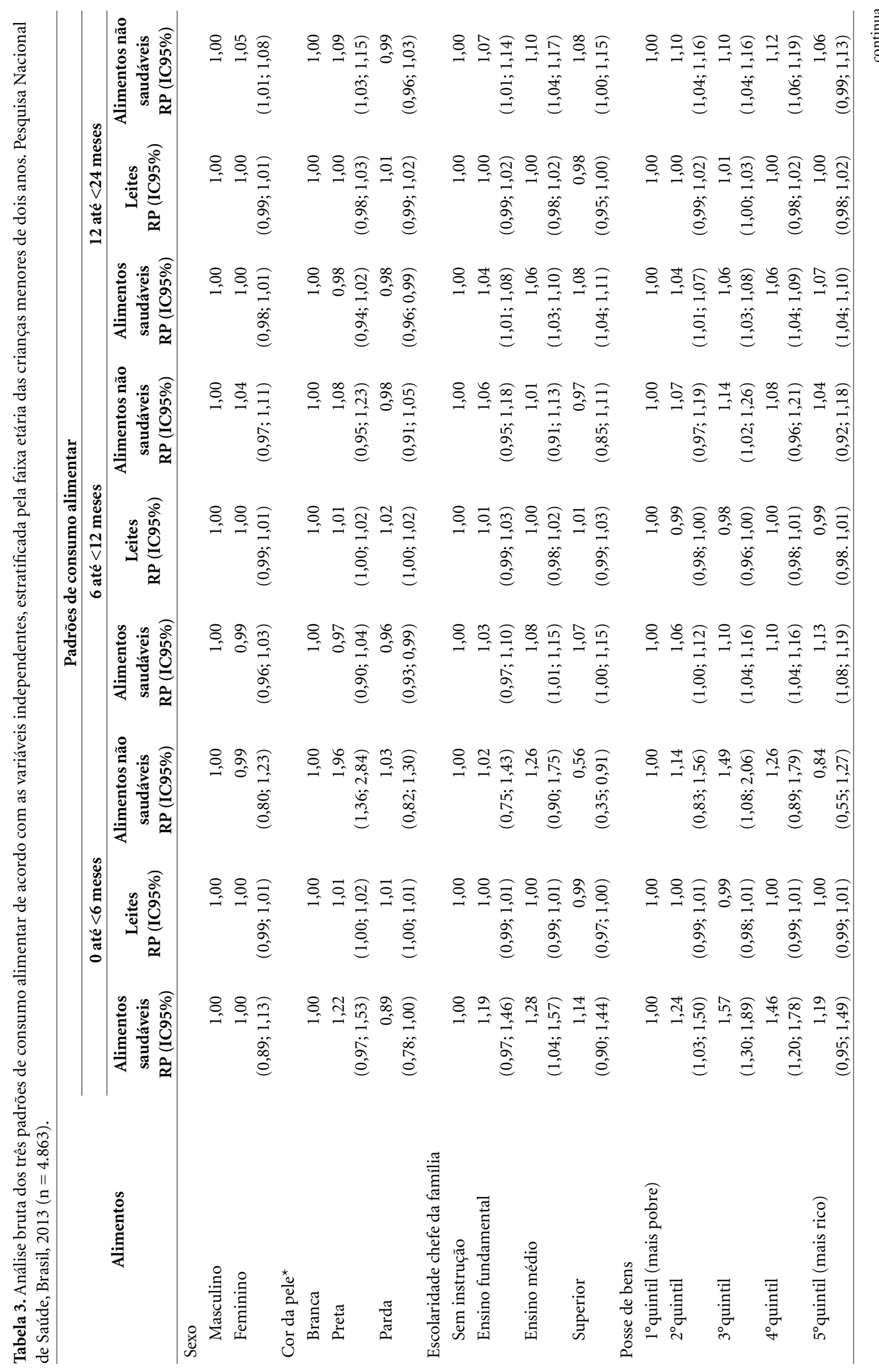




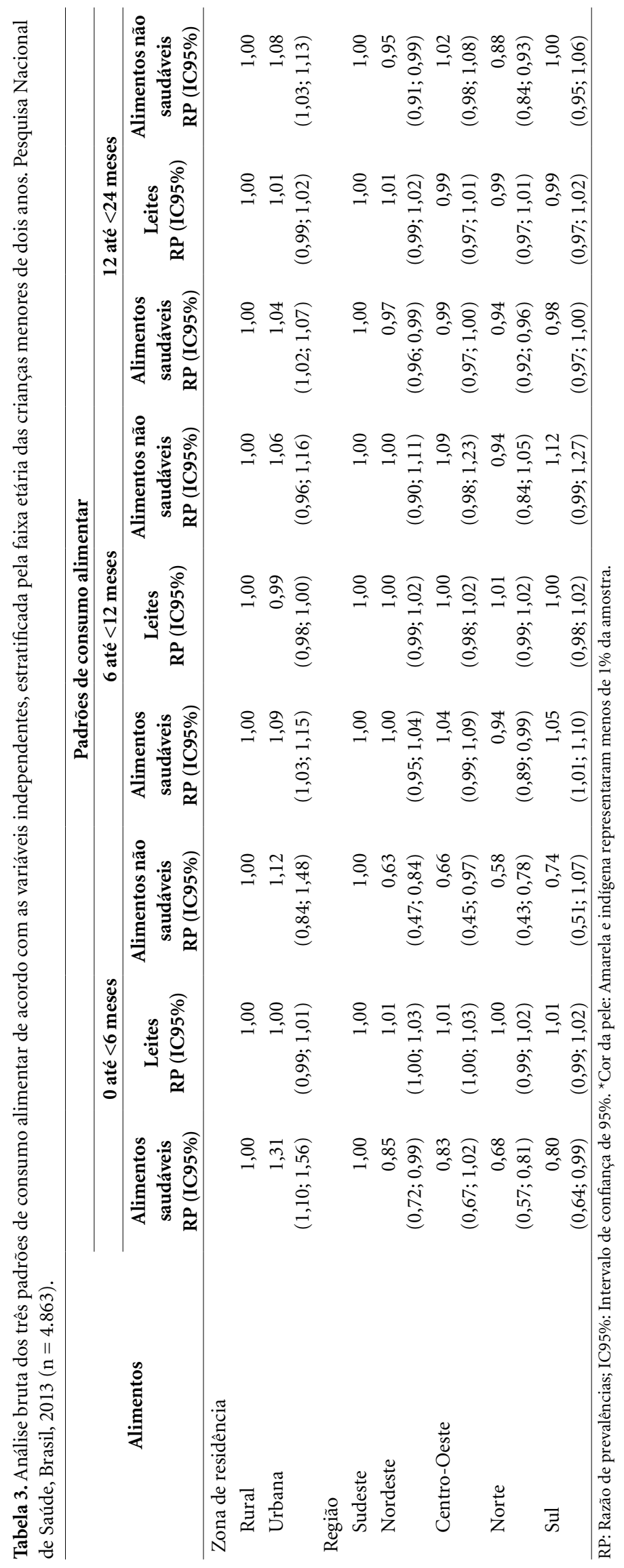




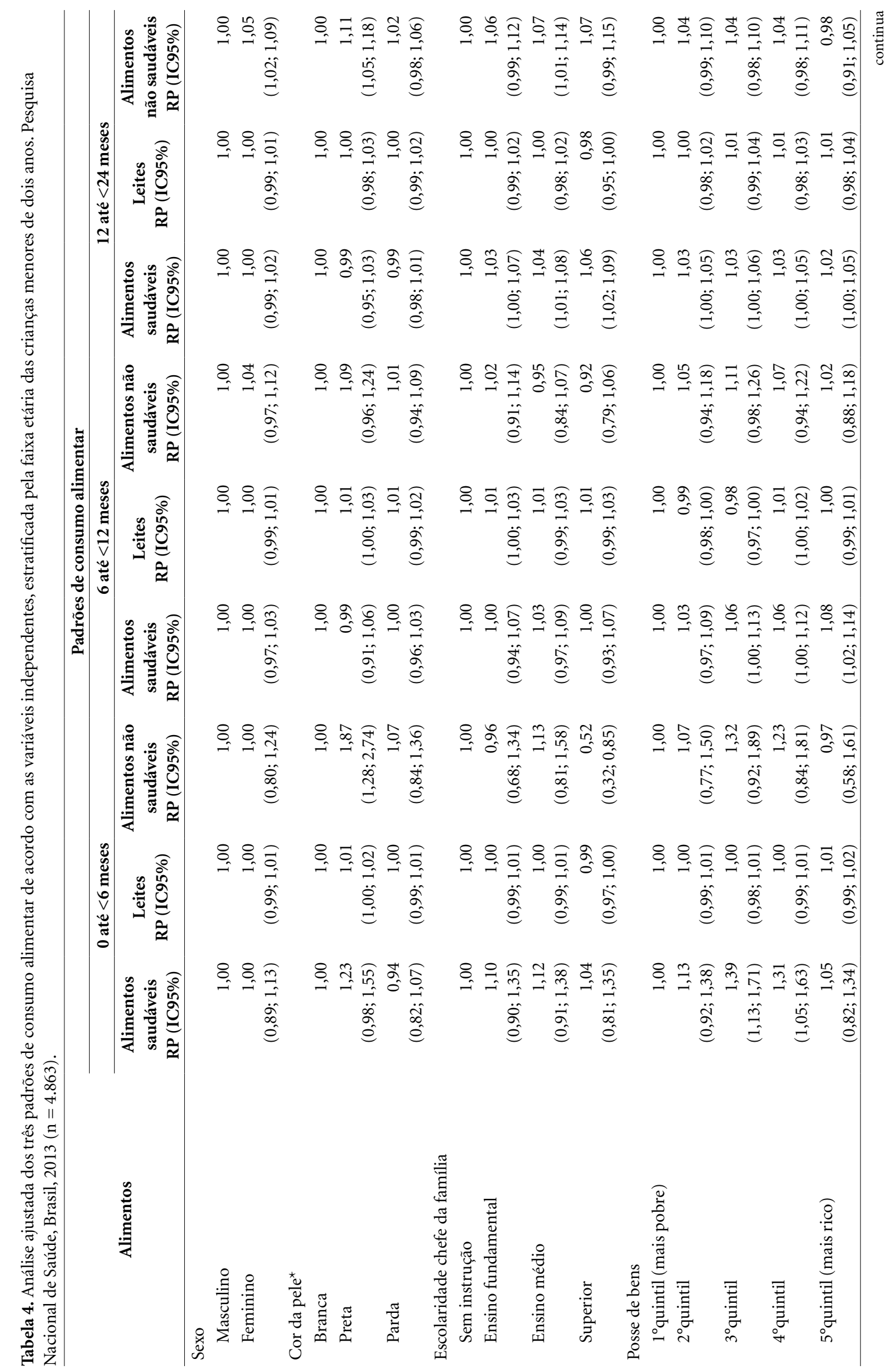


fato de ser em crianças menores de seis meses de idade, para as quais o preconizado ainda é apenas o leite materno ${ }^{1,26}$.

Os resultados da associação entre cor da pele preta e maior adesão ao padrão de consumo de alimentos não saudáveis, entre as crianças menores de seis meses de idade, se confirmam pela associação com a escolaridade do chefe da família onde crianças cujos chefes de família possuem ensino superior apresentaram menor probabilidade de aderir ao padrão não saudável da dieta. Nesse sentido, além do nível econômico dessas crianças, quanto maior a instrução dos indivíduos melhor será seus indicadores de saúde ${ }^{28}$, incluindo a alimentação. Um estudo sobre disponibilidade domiciliar encontrou que o nível de educação do chefe da família esteve associado a alta disponibilidade de alimentos, exceto de vegetais e refrigerantes ${ }^{23}$. Ainda, outro estudo realizado com crianças de um a seis anos de idade, observou-se que as condições socioeconômicas possuem forte influência no padrão alimentar das crianças, pois os padrões "vegetais" e "frutas", por exemplo, foram mais aderidos por crianças com maior renda familiar e cujas mães possuíam maior escolaridade ${ }^{15}$.

Ainda, entre as crianças menores de seis meses de idade observou-se que o oferecimento de alimentos pertencentes ao grupo "saudável" foi mais prevalente entre as pertencentes ao quintil mais pobre. Neste sentido, foi observado por outros estudos ${ }^{26,29}$ que amamentação, por exemplo, é mais frequente nos estratos considerados mais ricos da população. O mesmo padrão foi observado nas demais faixas etárias, quando se observou que tanto para escolaridade como para cor da pele o padrão de alimentos considerados não saudáveis é mais prevalente nos grupos mais suscetíveis. Esses achados enfatizam a necessidade de reforço nas estratégias já existentes ${ }^{4}$ que visam a educação alimentar da população, mas principalmente da parcela mais vulnerável.

A maior parte dos estudos que avaliam os padrões alimentares são realizados com crianças menores de cinco anos de idade, em idade escolar e com adolescentes ${ }^{6,7,13,30}$, mesmo que existam estudos com menores de dois anos de idade, com avaliações semelhantes à utilizada no presente estudo $^{14,31}$. Por isso, também, a comparabilidade dos achados do presente estudo pode ser considerada limitada. No entanto, pode-se considerar a falta de comparabilidade uma limitação inerente aos estudos de padrões alimentares em função dos diferentes instrumentos disponíveis para avaliação de consumo alimentar, principal- 
mente em crianças (Questionário de Frequência Alimentar do Sistema de Vigilância Alimentar e Nutricional, http://dabsistemas.saude.gov.br/sistemas/sisvan). Por outro lado, a utilização desta metodologia permite identificar padrões que representem a alimentação de cada população estudada, resultando em indicadores específicos e que refletem a realidade de cada amostra. Mesmo assim, os resultados deste estudo podem contribuir substancialmente para a literatura dessa temática entre os menores de dois anos de idade.

O presente estudo possui limitações. A mais forte seria a do recordátorio de 24 h que pode não representar o hábito alimentar da criança, no entanto, esse período é muito utilizado em estudos transversais epidemiológicos ${ }^{6,7,15}$ que avaliam consumo alimentar. Mesmo assim, acredita-se que os achados deste estudo sejam compatíveis com a realidade das crianças dessa faixa etária (menores de dois anos de idade), salientando a importância destes e sua contribuição para a literatura. Cabe ressaltar, também, os pontos fortes deste trabalho como a representatividade nacional da amostra e a avaliação dos padrões não só no período de seis meses, como também, até os 24 meses de idade, sendo considerada uma fase muito importante para o desenvolvimento das crianças e estabelecimento de intervenções efetivas $^{17}$.

Os padrões identificados neste estudo foram claros e não restritos somente a fase de alimentação complementar, conforme recomendação da OMS. Foi possível observar que uma série de alimentos foram oferecidos as crianças com menos de seis meses de idade. Mais de 35\% da amostra que, conforme o preconizado, deveria estar recebendo apenas leite materno, consumiu mingau, frutas e outros leites no dia anterior à entrevista. Esse resultado é preocupante, podendo refletir os vestígios culturais estabelecidos no Brasil. Embora todas as ações que salientam a importância do leite materno, da alimentação complementar adequada a partir dos seis meses de idade e de não oferecimento de alimentos açucarados e com alto teor de sal e gordura antes dos 24 meses de idade, os achados referentes aos hábitos alimentares que as crianças brasileiras estão formando é preocupante e com poucas evidências de melhora deste cenário. Os resquícios da transição nutricional ${ }^{32}$ que acometeu o país juntamente com a demográfica e epidemiológica acarretaram cargas que ultrapassaram gerações e, assim, estudos prospectivos são necessários para confirmar a existência de tais padrões nas faixas etárias estudadas.

\section{Colaboradores}

TR Flores idealizou o estudo, preparou o banco e analisou os dados, descreveu os resultados, participou na escrita do manuscrito completo. RG Neves auxiliou nas análises dos dados, na escrita dos métodos e discussão e revisou a versão final. CS Costa e A Wendt participaram de rodadas de revisão e da versão final do manuscrito. $\mathrm{AD}$ Bertoldi revisou a versão final com contribuições pertinentes. BP Nunes preparou o banco e auxiliou as análises dos dados e revisou a versão final do manuscrito.

\section{Agradecimentos}

Instituto Brasileiro de Geografia e Estatística e Ministério da Saúde pela realização da Pesquisa Nacional de Saúde.

\section{Referências}

1. World Health Organization (WHO). Complementary feeding: Report of the global consultation. Geneva: WHO; 2001.

2. Brasil. Ministério da Saúde (MS). Secretaria de Atenção à Saúde. Departamento de Atenção Básica. Saúde da criança: crescimento e desenvolvimento. Brasília: MS; 2012.

3. Brasil. Ministério da Saúde (MS). Secretaria de Atenção à Saúde. Departamento de Atenção Básica. Saúde da criança: nutrição infantil: aleitamento materno e alimentação complementar. Brasília: Editora do MS; 2009.

4. Brasil. Ministério da Saúde (MS). Secretaria de Atenção à Saúde. Departamento de Atenção Básica. Dez passos para uma alimentação saudável: guia alimentar para crianças menores de dois anos: um guia para o profissional da saúde na atenção básica. Brasília: MS; 2013

5. Garcia R. A comida, a dieta, o gosto: mudanças na cultura alimentar urbana [tese]. São Paulo: Universidade de São Paulo; 1999. 
6. Matos S, Barreto M, Rodrigues L, Oliveira V, Oliveira L, D'Innocenzo S, Teles CAS, Pereira SRS, Prado MS, Assis AMO. Padrões alimentares de crianças menores de cinco anos de idade residentes na capital e em municípios da Bahia, Brasil, 1996 e 1999/2000. Cad Saúde Pública 2014; 30(1):44-54.

7. Farias Júnior G, Osório M. Padrão alimentar de crianças menores de cinco anos. Rev Nutr 2005; 18(6):793802.

8. Mallarino C, Gómez L, González-Zapata L, Cadena Y, Parra D. Advertising of ultra-processed foods and beverages: children as a vulnerable population. Rev Saúde Pública 2013; 47(5):1006-1010.

9. Prado M, Assis A, Martins M, Nazaré M, Rezende I, Conceição M. Hipovitaminose A em crianças de áreas rurais do semiárido baiano. Rev Saúde Pública 1995; 29(4):295-300.

10. Assis A, Barreto M, Santos L, Sampaio L, Magalhães L, Prado MEA. Condições de vida, saúde e nutrição na infância em Salvador. São Paulo: Editora Bureau; 2000.

11. World Health Organization (WHO). Preparation and use of food basead dietary guidelines. Geneva: WHO; 1998.

12. D’Innocenzo S, Marchioni D, Prado M, Matos S, Pereira S, Barros AEA. Condições socioeconômicas e padrões alimentares de crianças de 4 a 11 anos: estudo SCAALA-Salvador-Bahia. Rev Bras Saúde Matern Infant 2011; 11(1):41-49.

13. Craig L, McNeill G, Macdiarmid J, Masson L, Holmes B. Dietary patterns of school-age children in Scotland: association with socio-economic indicators, physical activity and obesity. Br J Nutr 2010; 103(3):319-334.

14. Gatica G, Barros A, Madruga S, Matijasevich A, Santos I. Food intake profiles of children aged 12, 24 and 48 months from the 2004 Pelotas (Brazil) birth cohort: an exploratory analysis using principal components. Int J Behav Nutr Phys Act 2012; 9:43.

15. Souza R, Madruga S, Gigante D, Santos I, Barros A, Assunção M. Padrões alimentares e fatores associados entre crianças de um a seis anos de um município do Sul do Brasil. Cad Saúde Pública 2013; 29(12):24162426.

16. Flores T, Nunes B, Neves R, Wendt A, Costa C, Wehrmeister F, Bertoldi AD. Consumo de leite materno e fatores associados em crianças menores de dois anos: Pesquisa Nacional de Saúde, 2013. Cad Saúde Pública 2017; 33(11):e00068816.

17. Save the Children. Nutrition in the first 1,000 days state of the World's Mothers 2012. Fairfield: Save the Children; 2012.

18. Instituto Brasileiro de Geografia e Estatística (IBGE). Pesquisa Nacional de Saúde, PNS, 2013. Questionário dos moradores do domicílio [Internet]. [acessado 2018 Nov 17]. Disponível em: http://www.ibge.gov.br/ home/estatistica/populacao/pns/2013/

19. The Scree Test For The Number Of Factors. Multivariate Behavioral Res 1966; 1(2):245-276.

20. Norman G, Streiner D. Biostatistics: The Bare Essentials. McGraw-Hill Europe; 2008.
21. Barros A, Hirakata V. Alternatives for logistic regression in cross-sectional studies: an empirical comparison of models that directly estimate the prevalence ratio. BMC Med Res Methodol 2003; 3:21.

22. Popkin B, Adair L, Ng S. Global nutrition transition and the pandemic of obesity in developing countries. Nutr Rev 2012; 70(1):3-21.

23. Soares A, França G, Gonçalves H. Disponibilidade domiciliar de alimentos em Pelotas (RS): uma abordagem do ambiente obesogênico. Rev Nutr 2014; 27(2):193-203.

24. Martensen A, Grønholdt L. Children's influence on family decision making. Innov Mark 2008; 4(4):14-22.

25. Vereecken C, Haerens L, De Bourdeaudhuij I, Maes L. The relationship between children's home food environment and dietary patterns in childhood and adolescence. Public Health Nutr 2010; 13(10A):17291735.

26. Victora C, Bahl R, Barros A, França G, Horton S, Krasevec J, Murch S, Sankar MJ, Walker N, Rollins NC, Lancet Breastfeeding Series Group. Breastfeeding in the 21st century: epidemiology, mechanisms, and lifelong effect. Lancet 2016; 387(10017):475-490.

27. Victora C, Aquino E, Leal M, Monteiro C, Barros F, Szwarcwald C. Saúde de mães e crianças no Brasil: progressos e desafios. Lancet 2011; 2:32-46.

28. Paim J, Travassos C, Almeida C, Bahia L, Macinko J. O sistema de saúde brasileiro: história, avanços e desafios. Lancet 2011; Saúde no Brasil (1):11-31.

29. Victora C, Adair L, Fall C, Hallal P, Martorell R, Richter L, Sachdev HS, Maternal and Child Undernutrition Study Group. Maternal and child undernutrition: consequences for adult health and human capital. Lancet 2008; 371(9609):340-357.

30. Silva RCR, Assis AMO, Szarfarc SC, Pinto EJ, Costa LCC, Rodrigues LC. Iniquidades socioeconômicas na conformação dos padrões alimentares de crianças e adolescentes. Rev Nutr 2012; 25(4):451-461.

31. Bell L, Schammer C, Devenish G, Ha D, Thomson M, Spencer J, Do L, Scott J, Golley R. Dietary Patterns and Risk of Obesity and Early Childhood Caries in Australian Toddlers: Findings from an Australian Cohort Study. Nutrients 2019; 11(11):2828.

32. Batista-Filho M, Rissin A. A transição nutricional no Brasil: tendências regionais e temporais. Cad Saúde Pública 2003; 19(Supl. 1):S181-S191.

Artigo apresentado em 02/06/2020

Aprovado em 21/07/2020

Versão final apresentada em 23/07/2020

Editores chefes: Romeu Gomes, Antônio Augusto Moura da Silva 